\title{
Erythema Multiforme is Not Always Erythema Multiforme
}

\author{
Hana Nasim Ahmed, MD¹, Meagan Mandabach Olivet, BA ${ }^{1}$, Carly Elston, MD¹ \\ Boni Elewski MD
}

${ }^{1}$ The University of Alabama at Birmingham

\section{ABSTRACT}

Rowell syndrome (RS) describes the rare presentation of EM like lesions found in association with a diagnosis of lupus erythematosus (LE). Major diagnostic criteria includes: LE, EM, and anti-nuclear antibodies. Minor criteria includes: chilblains, anti-Ro/anti-La antibodies, and rheumatoid factor. We present a 26 -year-old white male seen in consultation for chronic erythema multiforme (EM) with a duration of 6 months unresponsive to treatment. Prior to our evaluation, he had been seen by another dermatologist and was diagnosed with biopsy-proven EM. His rash did not respond to previous treatments, including corticosteroids and antifungals. Lab results showed a positive ANA and positive anti-Ro antibody. The patient met diagnostic criteria for RS and was started on hydroxychloroquine $200 \mathrm{mg}$ twice daily. Improvement was noticed two weeks after beginning treatment. Our case demonstrates that an atypical presentation of recurrent erythema multiforme, which does not respond to typical EM treatment, should raise a suspicion for RS and prompt screening for autoimmune markers and lupus erythematosus.

\section{INTRODUCTION}

Rowell syndrome (RS) describes the rare presentation of EM like lesions found in association with a diagnosis of lupus erythematosus (LE). Major diagnostic criteria includes LE, EM, and anti-nuclear antibodies. Minor criteria includes: chilblains, anti-Ro/anti-La antibodies, and rheumatoid factor.

\section{CASE REPORT}

We present a 26-year-old white male seen in consultation for chronic erythema multiforme $(E M)$ with a duration of 6 months unresponsive to treatment. Prior to being seen in our clinic, he had been evaluated by another dermatologist and was diagnosed with biopsy-proven EM. In the following weeks, he received prednisone tapers, topical corticosteroids, oral antihistamines, valacyclovir, azithromycin, and topical antifungals, to which his rash did not respond. Preliminary lab work was also ordered, including a complete blood count (CBC), a comprehensive metabolic panel (CMP), and an antinuclear antibody (ANA). The ANA was positive, and he was pending a consult with rheumatology. Upon evaluation, he was nearly erythrodermic. On obtaining further history, the patient denied recent new medications, pneumonia or upper respiratory infection, herpes labialis, or genital herpes. On examination, the rash consisted of pink annular-to-polycyclic plaques with dusky centers and thin powdery scale that coalesced, mainly localized to his torso, back, and proximal upper and lower extremities with 60-70\% 
BSA involvement. His palms, soles, and mucous membranes were spared (Figure 1).



Figure 1

Additional lab work was obtained and included CBC, CMP, glucose-6-phosphate dehydrogenase, T-spot test, acute hepatitis panel, flow cytometry, a dermatomyositis panel, ANA, anti-Ro, and Anti-La. The labs were within normal limits except for a repeated positive ANA (1:320 with a speckled pattern) and positive anti-Ro antibody.

Punch biopsies were collected from three distinct anatomical locations, two were sent for hematoxylin and eosin (H\&E) staining and the other for direct immunofluorescence (DIF). The DIF was negative and H\&E staining indicated an interface dermatitis with prominent dyskeratotic cells appreciated at multiple levels of the epidermis, favoring an EM spectrum disorder (Figures 2 and 3). There was also increased mucin noted in the dermis. A deep inflammatory infiltrate involving adnexal structures was not appreciated.

Given the positive ANA and Anti-Ro, a diagnosis favoring Rowell Syndrome (RS) was made and the patient was started on hydroxychloroquine $200 \mathrm{mg}$ twice daily. After two weeks after initiation of this treatment, the patient began to note improvement of his rash.



Figure 2



Figure 3 


\section{DISCUSSION}

RS describes the rare presentation of EM like lesions found in association with a diagnosis of lupus erythematosus (LE). First described in the literature by Scholtz in 1922, the diagnostic criteria of LE, EM-like lesions, and positive rheumatoid factor and antinuclear antibodies was created by Rowell et al. in 1963. Zeitouni et al. proposed the major criteria of LE, EM, and anti-nuclear antibodies, and the minor criteria of chilblains, anti-Ro/anti-La antibodies, and rheumatoid factor. Our patient meets two of the major diagnostic criteria and fulfills the minor criterion of antiRo and anti-La antibodies. The majority of reports discuss adult female patients, making this report unique with an adult male patient. $^{1}$

It is important to note that subacute cutaneous lupus erythematosus (SCLE) can also appear as annular pink plaques on the trunk; however, upon examination on DIF, SCLE tends to show a granular deposition of $\lg G$ and $\operatorname{lgM}$ at the dermo-epidermal junction and sometimes within the epidermis. In general, though, a negative DIF does not necessarily rule out a diagnosis of cutaneous lupus. In our case, the DIF was negative and $\mathrm{H} \& \mathrm{E}$ staining indicated an interface dermatitis with prominent dyskeratotic cells appreciated at multiple levels of the epidermis, favoring an EM spectrum disorder. A thickened basement membrane zone or deep inflammatory infiltrate involving adnexal structures was not appreciated. Given the clinical and histological features, the rash was diagnosed as EM rather than SCLE.

RS is typically associated with acute cutaneous lupus erythematosus (ACLE). ${ }^{2}$ As noted in our case, a primary diagnosis of LE was not met. Though LE is a major diagnostic criterion of RS, another case report by Arevalo et al. documented a case of a 20 year-old male with no prior LE diagnosis. ${ }^{3}$ Clinicians evaluating an atypical presentation of EM should keep RS in mind regardless of a previous diagnosis of LE, refer their patients for the screening of LE, and order appropriate serology.

RS has been characterized by its absence of triggering factors, as in this case, distinguishing it from EM and LE. ${ }^{4,5}$ Because of this, a thorough history ruling out all known causes of EM, such as Herpes simplex or drug-induced, is imperative for a diagnosis of RS. Additionally, because of the use of immunosuppressant drugs, LE patients may be predisposed to infections which also can trigger EM. ${ }^{4}$ Brănişteanu et al. outlined a rare case in which RS occurred one week after beginning treatment for Helicobacter pylori. ${ }^{6}$ In a report by Ward et al., a patient's RS was attributed to sun exposure following a tapered dose of hydroxychloroquine and methotrexate, though photosensitivity is also characteristic of LE. ${ }^{7}$

Management for RS includes the use of steroids and immunosuppressive drugs, including azathioprine and cyclosporine.,3,7 Antimalarials, such as hydroxychloroquine and chloroquine, have also been employed, as well as dapsone. ${ }^{1,4,8,9}$ Our patient has responded well to hydroxychloroquine.

\section{CONCLUSION}

RS is a rare and complex diagnosis requiring a thorough history and screening. As our case demonstrates, an atypical presentation of recurrent erythema multiforme which does not respond to typical EM treatment should raise a suspicion for 
RS and prompt screening for autoimmune markers and lupus erythematosus.

\section{Conflict of Interest Disclosures: None}

Funding: None

\section{Corresponding Author:}

Meagan Mandabach Olivet, B.A.

The University of Alabama at Birmingham

Email: mkmandab@uab.edu
9. Lee A, Batra P, Furer V, Cheung W, Wang N, Franks A, Jr. Rowell syndrome (systemic lupus erythematosus + erythema multiforme). Dermatol Online J. Aug 15 2009;15(8):1.

10. Zeitouni NC, Funaro D, Cloutier RA, Gagne E, Claveau J. Redefining Rowell's syndrome. Br J Dermatol. Feb 2000;142(2):343-6. doi:10.1046/j.1365-2133.2000.03306.x

11. Bolognia JL, Schaffer JV, Cerroni L, Callen JP. Chapter 41 Lupus Erythematosus. In: Dermatology. Edinburgh: Elsevier; 2018.

\section{References:}

1. Gallo L, Megna M, Festa B, et al. Rowell Syndrome: A Diagnostic Challenge. J Clin Aesthet Dermatol. Apr 2020;13(4):40-42.

2. Okon LG, Werth VP. Cutaneous lupus erythematosus: diagnosis and treatment. Best Pract Res Clin Rheumatol. Jun 2013;27(3):391-404. doi:10.1016/j.berh.2013.07.008

3. Arevalo AB, Nassar R, Krishan S, Lakshmanan P, Salgado M, Chokshi P. Lupus Never Fails to Deceive US: A Case of Rowell's Syndrome. Case Rep Rheumatol. 2020;2020:8884230. doi: $10.1155 / 2020 / 8884230$

4. Anuj Sharma RS. Rowell Syndrome: A case report and review of literature'. Internet Journal of Rheumatology and Clinical Immunology. 1 September 2016 2016;4(1)

5. Devang Solanki ED, Nishita Drji. Case Report of a Rowell's Syndrome. International Journal of Science and Research. 2014;3(1):7-8.

6. Branisteanu DE, lanosi SL, Dimitriu $A$, Stoleriu G, Oanta A, Branisteanu DC. Druginduced Rowell syndrome, a rare and difficult to manage disease: A case report. Exp Ther Med. Jan 2018;15(1):785-788. doi:10.3892/etm.2017.5557

7. Callen JP, Klein J. Subacute cutaneous lupus erythematosus. Clinical, serologic, immunogenetic, and therapeutic considerations in seventy-two patients. Arthritis Rheum. Aug 1988;31(8):1007-13. doi:10.1002/art.1780310811

8. Muller CS, Hinterberger LR, Vogt T. Successful treatment of Rowell syndrome using oral cyclosporine A. Int J Dermatol. Aug 2011;50(8):1020-2. doi:10.1111/j.13654632.2010.04848.x 